\title{
江淮梅雨期各类持续性降水频数年代际尺度上的变异及 其可能原因的探讨
}

\author{
黄丹青, 朱坚, 况雪源 \\ 南京大学大气科学学院, 南京大学灾害性天气气候研究所, 南京 210093 \\ E-mail: huangdq@nju.edu.cn \\ 2010-07-23 收稿, 2010-10-11 接受 \\ 公益性行业(气象)科研专项(GYHY200906015)、国家科技支撑计划(2009BAC51B03)和国家自然科学基金青年科学基金(40901016,40805041) \\ 资助
}

\begin{abstract}
摘要 围绕 1960 2007 年江淮地区梅雨期各类持续性降水, 展开了各类持续性降水年代际变 异的研究, 并从低层大气季节内扰动的角度, 着重探讨了持续 $5 \mathrm{~d}$ 及以上降水频数年代际变 异的特征及可能原因. 研究发现, 气候平均而言, 随着持续时间的增加, 总频数逐渐减少, 并且频发区从江淮的北部地区逐步向南部地区移动. 各类持续性降水频数年代际趋势揭示 出 2000 年后长持续时间降水明显减少的事实, 随之增加的是位于长江流域的持续 $2 \mathrm{~d}$ 的降水 过程以及长江以北地区的持续 3 4 $\mathrm{d}$ 的降水. 同时发现, 低层风场季节内扰动的增加和减少 直接与持续较长时间降水频数的年代际变异相关, 尤其是在 2000 年后, 季节内扰动信号明显 减弱, 持续时间较长的单峰低频信号逐渐被持续时间较短的多峰结构所取代, 从而导致近年 来(2000 2007 年)长持续时间降水显著减少. 并且, 低层斜压性的年代际差异也与长持续性降 水频数的异常相吻合. 近年来, 江淮地区斜压性明显减弱也为持续 $5 \mathrm{~d}$ 及以上降水的减少提 供有利的背景场.
\end{abstract}

\section{关键词}

江淮梅雨

各类持续性降水

季节内振荡

低层斜压性
江淮梅雨是春末夏初东亚夏季风季节进程中特 有的雨季, 也是夏季影响我国东部的重要天气气候 现象. 梅雨的强弱、梅雨期的长短以及梅雨量的多寡 等特征既反映了亚洲上空大气环流季节变化与环流 调整的各种演变过程, 而且直接与江淮地区旱涝的 形成和持续性相关. 梅雨气候的异常变化一直是江 淮地区气候及气候变化研究的重要课题之一.

我国气象学家在梅雨年代际尺度上的突变特征 方面做了大量工作. 魏凤英等人 ${ }^{[1]}$ 和陈艺敏等人 ${ }^{[2]}$ 对 1885 2000 年梅雨参数的突变情况进行检测, 他们的 结果均表明, 100 多年来, 梅雨长度和出梅日期发生 了气候突变, 除了梅雨参数的突变, 梅雨雨带在年际 以及年代际尺度上也存在振荡. 徐卫国等人 ${ }^{[3]}$ 利用我 国东部 180 个测站的逐日降水资料, 研究了 1961
2000 年梅雨期雨区的年代际变化, 并认为雨区边界 及雨区面积指数在 20 世纪 70 年代末 80 年代初出现 了突变. $\mathrm{Wu}$ 等人 ${ }^{[4]}$ 对比中国、日本和韩国台站雨量资 料, 结果表明, 中国东南部-长江中下游-中国东北部 雨型在 1977 1978 年发生转折. 徐群 ${ }^{[5]}$ 认为 1885 2000 年长江中下游梅雨期在 70 年代末发生了一次强 年代际突变, 即从 1958 1978 年的弱梅雨时期突变为 1979 1999 年的强梅雨时期, 且两时期的出梅期由偏 早转为延迟; 但从 2000 年开始, 长江中下游梅雨又 转人新的偏少期. 近年来, 不少学者提出梅雨新的变 化趋势, 自 2000 年以后, 梅雨期降水越来越集中, 集 中雨区偏向位于 $33^{\circ} \mathrm{N}$ 以北的淮河流域 ${ }^{[6 ~ 8]}$.

以往的研究中较多地关注梅雨参数、梅雨量和雨 带在年代际尺度上的突变. 然而, 持续性降水是梅 
雨期降水的重要特征, 并且, 持续长时间的降水易于 在该地区造成旱涝灾害, 那么, 各类持续性降水在不 同年代的演变特征是怎样的? 是否持续时间较长的 降水频数在 2000 年后显著下降? 如果是的话, 又是 什么原因导致了长持续性降水减少呢? 目前, 对梅 雨期各类持续性降水的研究较少, 希望搞清楚各类 持续性降水的演变特征, 尤其是持续时间较长的降 水变化情况, 这对理解 2000 年后梅雨新的变化趋势 和进一步认识梅雨“非典型变化”具有一定的意义.

\section{1 资料与方法}

本文选用的资料为中国气象局整编的全国 738 个测站 1960 年 1 月 2007 年 12 月共 $48 \mathrm{a}$ 的逐日降水 资料. 挑选出江淮地区 $\left(28^{\circ} \sim 34^{\circ} \mathrm{N}, 110^{\circ} \sim 122^{\circ} \mathrm{E}\right)$ 的站 点. 由于 $6 \sim 7$ 月的旱涝灾害大部分是由梅雨量异常所 引起的, 因此, 本文以江淮地区 $6 \sim 7$ 月的梅雨期作为 研究对象.

在分析各类持续性降水特征时，首先将持续性 降水细化分为持续 2, 3, 4, 5, $6 \mathrm{~d}$ 等 10 类, 进一步对 比分析发现某些持续性降水特征较类似(图略), 因 此, 最终选取持续 2, 3 4, $5 \mathrm{~d}$ 及以上共 3 种持续性降 水的类型. 各类持续性降水均不重复计算, 比如, 持 续 3 4 d 的降水中并不包括持续 $2 \mathrm{~d}$ 的情况. 将出现 各类持续性降水过程的次数定义为各类持续性降水 的频数.

本文选用的大气资料取自 NCEP/NCAR(National
Centers for Environmental Prediction/National Center for Atmospheric Research)再分析资料中的常规气象 变量的逐日数据集, 该资料的经纬网格为 $2.5^{\circ} \times 2.5^{\circ}$, 时间长度为 $63 \mathrm{a}$,从 1948 年 1 月至 2010 年 1 月. 主 要选用的要素场为 850 和 $700 \mathrm{hPa}$ 的纬向风速、经向 风速和温度. 由于 NCEP/NCAR 再分析资料在 1979 年左右的低层资料还存在一定的不确定性 ${ }^{[9 \sim 12]}$, 因 此, 为了验证其结果, 本文还选取 ECMWF(European Center for Medium-Range Weather Forecasts)的再分 析资料 ERA40 作对比. 该资料的经纬网格为 $2.5^{\circ} \times 2.5^{\circ}$, 时间长度为 $45 \mathrm{a}$, 从 1957 年 9 月 2002 年 8 月.

采用 Hamming ${ }^{[13]}$ 介绍的带通滤波技术, 从 NCEP/ NCAR 和 ERA40 逐日资料中滤出 30 60 d 的季节内 振荡的扰动信号, 选取逐年的梅雨期逐日资料来代 表该年的梅雨期季节内扰动的信号. 同时, 引入最大 扰动增长速率(Maximum Eady growth rate $)^{[14,15]}$ 用于 比较低层大气斜压性的特征, 数值的大小反映斜压 性的强弱.

\section{2 结果与分析}

\section{1 各类持续性降水的总频数}

图 1 给出各类持续性降水在 1960 2007年的总频 数分布. 由图 1(a)得知, 江淮地区持续 $2 \mathrm{~d}$ 降水的总 频数都在 100 次以上, 南部地区出现持续 $2 \mathrm{~d}$ 降水的 频数比北部少, 频发区位于江淮的偏北地区. 图 1(b) 和(c)分别为持续 $3 \sim 4 \mathrm{~d}$ 和持续 $5 \mathrm{~d}$ 及以上降水的 $48 \mathrm{a}$



图 $1 \quad 1960 \sim 2007$ 年共 $48 \mathrm{a}$ 各类持续性降水总频数分布

单位: 次数. 阴影区分别为持续 $2 \mathrm{~d}$ 降水大于 130 次(a); 持续 $3 \sim 4 \mathrm{~d}$ 降水大于 110 次(b); 持续 $5 \mathrm{~d}$ 及以上降水大于 70 次(c) 
总频数分布，随着持续日数的增加，各类持续性降水 总频数逐渐减少. 频发区分别位于江淮地区的中部、 长江三角洲地区和长江下游的南部地区. 比较 3 类持 续性降水频数，明显看出，随着持续时间的增加，总 频数逐渐减少, 并且频发区从江淮的北部地区逐步 向南部地区移动.

\section{2 各类持续性降水的均方差}

以往研究表明，在 1979/1980 年梅雨雨带位置及 降水量发生明显的突变 ${ }^{[16 ~ 21]}$, 最新的研究提出 2000 年后梅雨出现新的变化趋势 ${ }^{[5 \sim 8]}$. 为了比较年代际尺 度上的差异, 尤其是 2000 年后各类持续性降水的特 征. 因此, 以下将研究时段分为 1960 1979，1980 1999 和 2000 2007 年3 个时期, 分别比较不同时期内 各类持续性降水的变异特征.

对总频数的分析, 得到 3 类不同持续性降水的总 体分布. 但是，年际之间是否存在波动，不同时期的 波动幅度又有何差异? 通过对均方差的分析, 可知 该变量的离散程度, 即逐年之间的差异. 因此, 以下 给出各类持续性降水在不同时期的均方差分布.
随着时间的增长，持续 $2 \mathrm{~d}$ 降水的均方差大值中 心(图 2(a), (d), (g)) 分别位于江淮的东北、西北和东南 地区. 表明, 在上述地区，持续 $2 \mathrm{~d}$ 降水的频数年际 之间差异较大, 某些年份次数较多, 某些年份则较 少. 持续 3 4 d 降水频数(图 2(b),(e),(h))年际之间的 离散程度在江淮南部的大部分地区均较大, 尤其是 在 1960 1979 年. 而在 2000 2007 年，江淮地区的持 续 3 4 d 降水频数年际之间差异较小. 持续 $5 \mathrm{~d}$ 及以 上的降水频数在 1960 1979 年(图 2(c))和 2000 年后 (图 2(i))的均方差大值区均在江淮的华南地区, 尤其 是 2000 年后, 即该地区某些年份频数较多, 某些较 小. 然而在 1980 1999 年(图 2(f)), 年际变化的大值 区位于江淮的东北地区.

\section{3 各类持续性降水的线性趋势}

图 3 为 1960 1979年各类持续性降水频数的显著 线性趋势分布. 持续 $2 \mathrm{~d}$ 降水(图 3(a)) 的显著趋势发 生在长江沿岸的站点, 并且均为显著线性上升趋势 持续 3 4 $\mathrm{d}$ 降水的显著线性趋势 (图 3(b)) 主要集中在 $118^{\circ} \mathrm{E}$ 以东的地区, 并且约以 $30^{\circ} \mathrm{N}$ 为界限, 以南为显
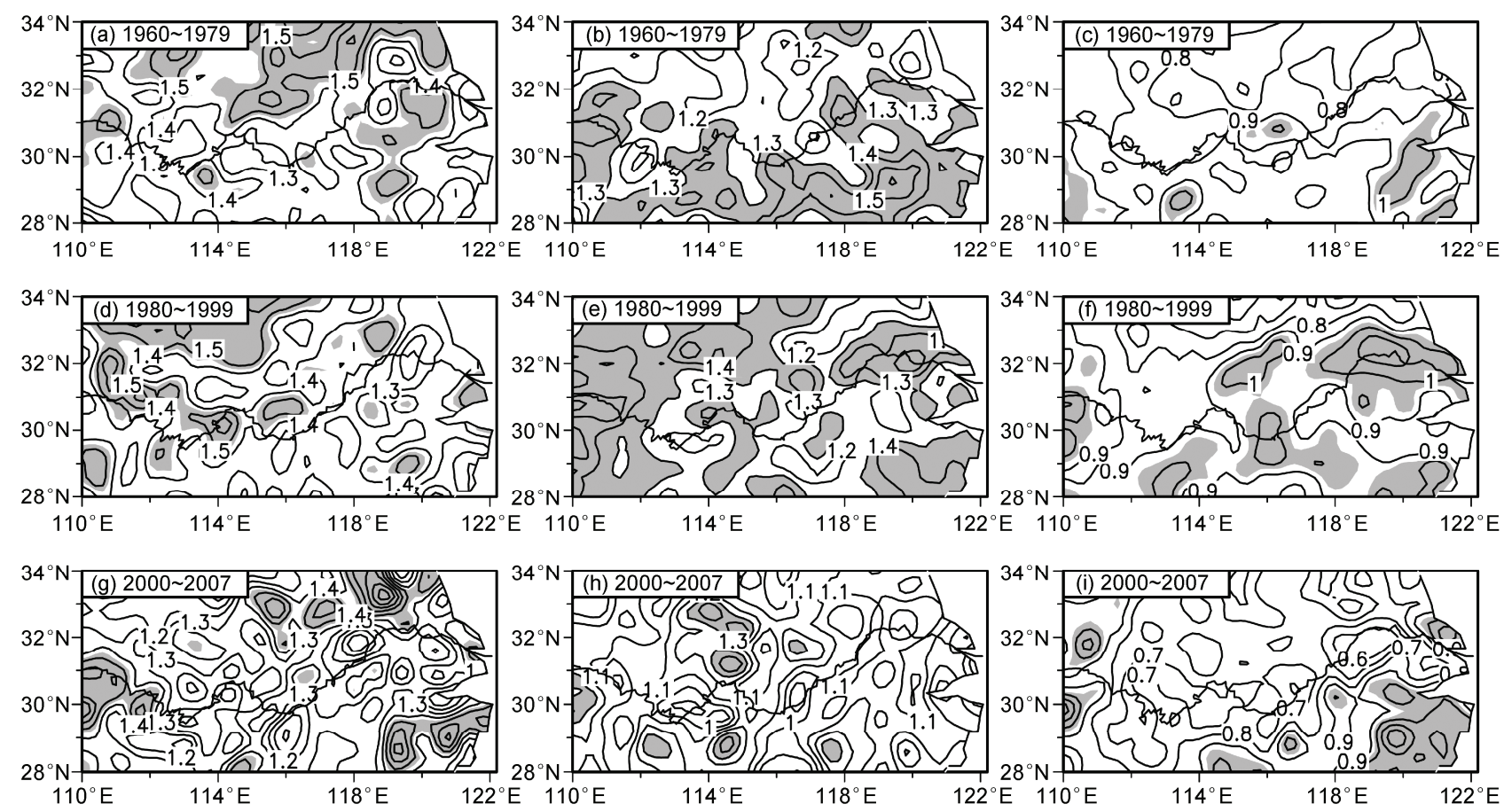

图 2 不同年代的各类持续降水频数的均方差分布

阴影区为大于 1.45((a),(d),(g)); 大于 1.3((b),(e),(h)); 大于 0.95((c),(f),(i)). (a), (d), (g) 为持续 $2 \mathrm{~d}$ 降水; (b),(e),(h)为持续 3 4 d 降水;

(c),(f),(i)为持续 $5 \mathrm{~d}$ 及以上降水 



$$
\begin{aligned}
& \triangle>0.02 \\
& \Delta 0.01 \sim 0.02 \\
& \Delta 0 \sim 0.01 \\
& \nabla-0.01 \sim 0 \\
& \nabla-0.02 \sim-0.01 \\
& \nabla<-0.02
\end{aligned}
$$

图 $31960 \sim 1979$ 年各类持续降水频数线性趋势分布

单位: 次数/a. 图中只给出通过 $95 \%$ 置信度检验的显著线性趋势的站点. (a) 持续 $2 \mathrm{~d}$ 降水; (b) 持续 3 4 d 降水; (c) 持续 $5 \mathrm{~d}$ 及以上降水

著上升趋势, 以北则为显著下降趋势. 持续 $5 \mathrm{~d}$ 及以 上降水的频数(图 3(c)) 为明显的南北反相变化, 在长 江以南地区为显著的线性上升趋势, 而江淮西北地 区则为较弱的线性减少趋势.

在 1980 1999 年(图 4), 各类持续性降水频数线 性趋势差异显著. 持续 $2 \mathrm{~d}$ 降水(图 4(a))的显著趋势 均为线性减少趋势, 并且, 在长三角地区尤为显著. 持续 3 4 d 降水(图 4(b))除了在湖南省北部地区为显 著上升趋势以外, 其他显著趋势均为线性下降趋势. 整体趋势呈现出从南到北的“+-+”的分布形式. 图 4(c) 为持续 $5 \mathrm{~d}$ 及以上降水频数的线性趋势与 1960 1979 年的较为一致, 主要表现在以长江为界限 的南北反相变化上, 长江以南大面积地区显著增加, 而偏北地区显著减少.

在 2000 2007 年, 持续 $2 \mathrm{~d}$ 降水(图 5(a)), 呈现出 西南往东北走向的线性上升趋势带, 并且显著的线 性上升趋势在长三角地区达到极大值. 而持续 3 4 d 的降水(图 5(b)), 显著趋势的站点主要集中在 $116^{\circ} \mathrm{E}$ 以东地区, 并且大部分站点为显著上升趋势, 而在江 淮偏北地区为线性下降趋势. 持续 $5 \mathrm{~d}$ 及以上降水频 数(图 5(c))的趋势主要表现为长江以南地区的显著线 性下降趋势, 降水显著增加区域转移至长江以北的 部分地区. 那么, 这类持续降水过程在长江以北增多 而长江以南地区则减少, 这也与司东等人 ${ }^{[7]}$ 最新的研 究结果梅雨雨带北移相吻合. 说明在 2000 2007 年 间，梅雨期持续 $5 \mathrm{~d}$ 及以上的降水过程在该地区显著 减少. 然而, 在梅雨期内往往存在一个或几个日照
少、湿度大的“连阴雨期”, 并且持续时间较长的降水 过程较多 ${ }^{[22]}$. 我们的研究表明, 近年来(2000 2007 年), 持续时间较长的降水过程在长江以南地区明显 减少，随之增加的则是持续 $2 \mathrm{~d}$ 和持续 $3 \sim 4 \mathrm{~d}$ 等持续 时间较短的突发性降水事件, 这与持续性降水较多 的典型梅雨特征并不一致, 也为近期一些研究中所 提出的梅雨“非典型变化”提供相应的佐证 ${ }^{[5 \sim 8]}$.

\section{3 与低层低频信号的可能联系}

通过对各类持续性降水不同时期差异的比较得 知, 各类持续性降水呈现出显著的年代际变化特征, 尤其是持续 $2 \mathrm{~d}$ 和持续 $5 \mathrm{~d}$ 及以上的降水频数, 主要 特征为持续 $5 \mathrm{~d}$ 及以上的降水频数在 1980 1999 年显 著增长，而之后(2000 2007 年)长持续时间的降水频 数明显减少. 这些分析结果都表明梅雨期持续性时 间较长的降水在 2000 年后出现明显的递减趋势, 尤 其是长江出海口附近的站点. 那么, 究竟是什么原因 导致持续性降水年代际变异，尤其是持续时间较长 的降水频数在 2000 年前后变化迥然不同? 较多的研 究揭示出持续性的强降水过程往往与大气的低频信 号关系密切, 尤其是低层的低频扰动信号 ${ }^{[23 ~ 30]}$. 本 文试图从低层的大气季节内扰动的角度来探讨与江 淮地区长时间持续性降水年代际异常的可能联系.

首先，不同时期的气候背景态之间存在差异，而 在变化后的气候背景态下, 各类持续性降水也随之 发生改变. 大气斜压性反映大气中不均匀加热的状 态, 体现大气不稳定性的强弱, 进而影响降水过程. 

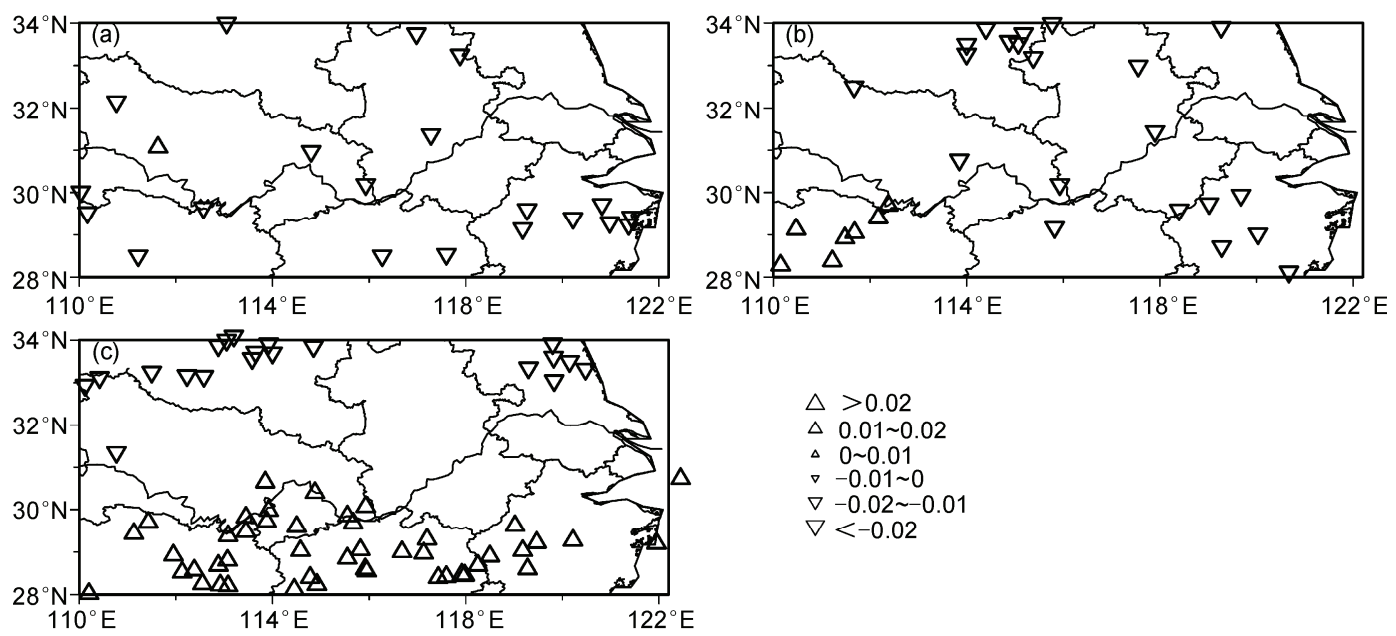

$$
\begin{array}{ll}
\triangle & >0.02 \\
\Delta & 0.01 \sim 0.02 \\
\Delta & 0 \sim 0.01 \\
\nabla & -0.01 \sim 0 \\
\nabla & -0.02 \sim-0.01
\end{array}
$$

图 4 1980 1999 年各类持续降水频数线性趋势分布

单位: 次数/a. 图中只给出通过 $95 \%$ 置信度检验的显著线性趋势的站点. (a) 持续 $2 \mathrm{~d}$ 降水; (b) 持续 3 4 d 降水; (c) 持续 $5 \mathrm{~d}$ 及以上降水
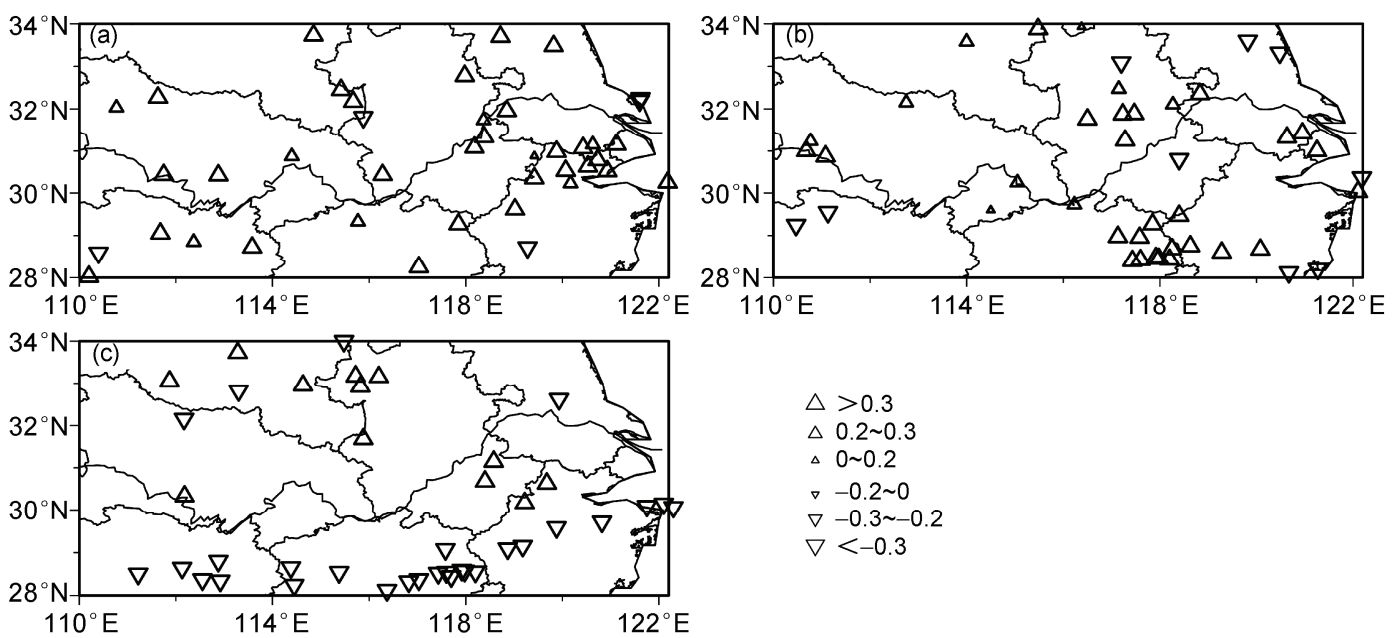

$$
\begin{aligned}
& \Delta>0.3 \\
& \Delta 0.2 \sim 0.3 \\
& \Delta 0 \sim 0.2 \\
& \nabla-0.2 \sim 0 \\
& \nabla-0.3 \sim-0.2 \\
& \nabla<-0.3
\end{aligned}
$$

图 $5 \quad 2000 \sim 2007$ 年各类持续降水频数线性趋势分布

单位: 次数/a. 图中只给出通过 95\%置信度检验的显著线性趋势的站点. (a) 持续 $2 \mathrm{~d}$ 降水; (b) 持续 3 4 $\mathrm{d}$ 降水; (c) 持续 $5 \mathrm{~d}$ 及以上降水

图 6 为不同时期的梅雨期低层最大扰动增长速率的差 值分布. 图中显示, 1980 1999 年与 1960 1979 年相 比(图 6(a)), 在中高纬地区, 东西向呈现出明显的正 负异常偶极子型, 而在江淮地区斜压性显著增强, 这 有利于该时期降水的增加. 然而将 2000 2007 年和 1980 1999 年相比较(图 6(b)), 在中高纬地区, 出现 南北向的负正异常偶极子, 负异常区域延伸至江淮 地区, 这也与江淮地区持续性较长的降水在近年来 明显减少相对应。

以上的分析发现，大背景态在不同时期的差异 不利于维持近年来持续性较长的降水, 那么, 从不同
时期的梅雨期低层风场的逐日演变中, 是否也能得 以验证? 图 7 给出不同时期, 梅雨期低层低频全风速 的逐日演变. 图中显示, 在 NCEP/NCAR 再分析资料 中(图 7(a)), 1960 1979 年, 低频全风速约在 6 月底 7 月中旬左右, 出现一个持续时间较长的峰值, 而该峰 值对应着持续时间较长的降水过程. 在 1980 1999 年, 分布与 1960 1979 年较类似, 但是该峰值出现的 时间则略有推迟. 然而, 近年来, 持续时间较长的低 频风场的单峰结构已被持续时间较短的多个峰值所 取代，并且持续性的降水过程发生时间也有所提前. 这也与最新的一些研究结果相吻合 ${ }^{[5]}$, 同样也验证, 



图 $61980 \sim 1999$ 年平均与 1960 1979 年平均(a)和 2000 2007 年平均与 1980 1999 年平均 $(b)$ 的梅雨期低层 $(850 \mathrm{hPa})$ 最大扰 动增长速度的差值

单位: $10^{6} \mathrm{~d}^{-1}$. 阴影区表示通过 $90 \%$ 信度检验的区域

近年来, 持续时间较长的降水过程相比于前两个时 期明显减少. 在 ERA40 再分析资料中(图 7(b)) 也能得 到一致的结论.

为了进一步分析年代际演变, 图 8 给出 1960 2007 年梅雨期江淮流域纬向和经向平均的持续 $5 \mathrm{~d}$ 及以上降水频数距平的时间剖面图. 从图 8(a)中可以 清楚地看出, 江淮流域持续 $5 \mathrm{~d}$ 及以上降水频数在 1960 1965 年减少, 并且减少的雨带从 $28^{\circ} \mathrm{N}$ 一直延 续至 $33^{\circ} \mathrm{N}$, 之后在 1970 1980 年, 持续 $5 \mathrm{~d}$ 及以上降 水频数增长, 增长的区域主要集中在 $30^{\circ} \sim 33^{\circ} \mathrm{N}$. 另 外发现, 在 1990 2007 年为持续 $5 \mathrm{~d}$ 及以上降水频数 变化剧烈的阶段, 其中, 在 1990 2000 年, 江淮流域
的长持续性降水频数剧烈增长, 主要的增长雨带位 于 $32^{\circ} \mathrm{N}$ 以南的地区，而之后(2000 2007 年)明显减 少, 并且主要的雨带与 1990 2000 年的较一致. 同时 发现，除了长持续时间的降水频数在不同时期的增 减之外, 异常的雨带也出现南北移动. 除了纬向变
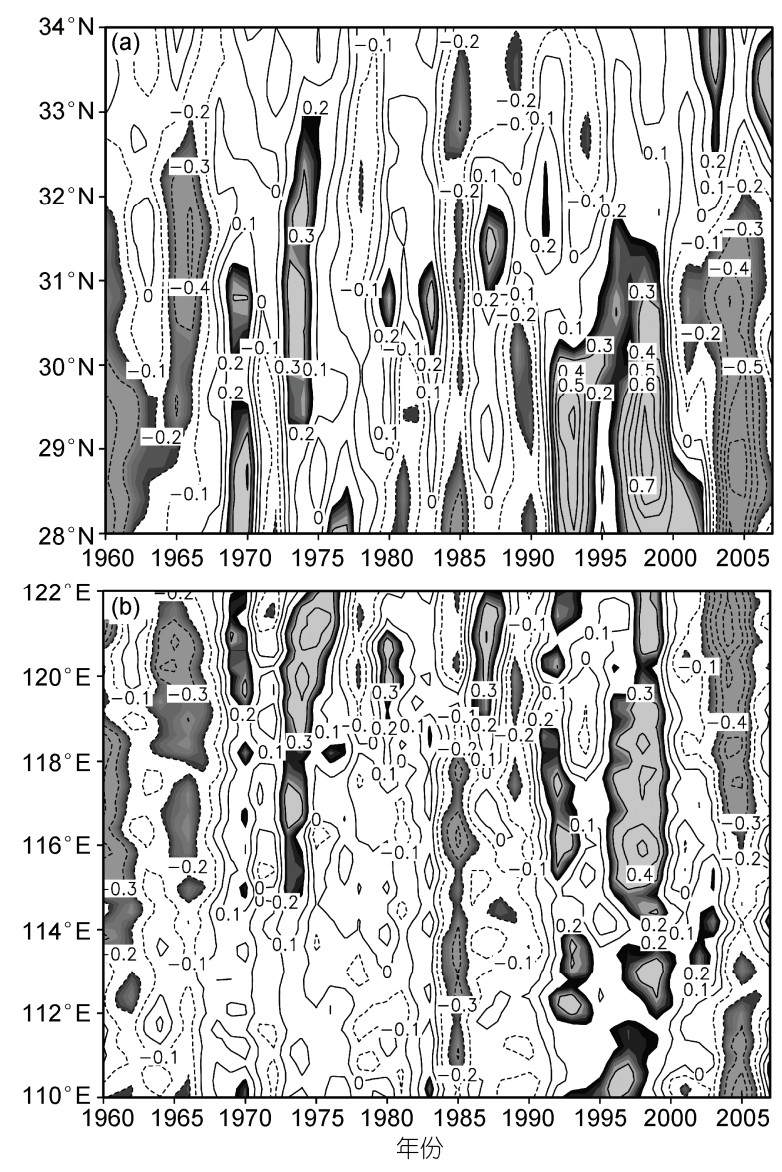

图 $81960 \sim 2007$ 年梅雨期 $110^{\circ} \sim 122^{\circ} \mathrm{E}$ 平均 $(\mathrm{a}) 、 28^{\circ} \sim 34^{\circ} \mathrm{N}$ 平均(b)的 $5 \mathrm{~d}$ 以上降水频数距平的时间-纬度、经度剖面图 (单位: 次数)


图 7 不同时期梅雨期低层低频全风速的逐日演变(单位: $\mathbf{m} / \mathbf{s}$ ) 
化, 东西向上, 也能得到类似的结果(图 8(b)), 并且, 随着时间的推移, 长持续性降水的频数变化也出现 由东向西的延伸.

图 9 为 1960 2007 年梅雨期江淮流域 $850 \mathrm{hPa}$ 的 低频全风速距平经向平均的时间剖面图. 在 NCEP/ NCAR 再分析资料中(图 9(a)), 低频信号的正负异常 中心与持续 $5 \mathrm{~d}$ 以上降水频数对应的较一致, 尤其是 在 1990 2007 年. 在 1992 2000 年, 低频信号和长持 续时间的降水频数均为显著正异常, 尤其是在 $20^{\circ} \sim 35^{\circ} \mathrm{N}$ 的纬度带内; 而近年来, 低频全风速距平 为显著负异常, 这也与持续 $5 \mathrm{~d}$ 以上降水频数异常相 对应. ERA40 再分析资料中(图 9(b)), 同样能抓住 90 年代的正异常信号, 异常中心延伸至 $35^{\circ} \mathrm{N}$, 在 2000


图 9 梅雨期 $110^{\circ} \sim 122^{\circ} \mathrm{E}$ 平均的低层季节内振荡的全风速距 平的时间-纬度剖面图

单位: m/s, (a) NCEP/NCAR, 1960 2007 年; (b) ERA40, 1960 2002 年
年后, 负异常信号出现在 $25^{\circ} \sim 40^{\circ} \mathrm{N}$ 的纬度带内, 这 都与持续较长的降水频数演变相吻合. 从低频全风 速的纬向平均的时间-经度剖面图中(图略), 也发现 类似的结论: 低频信号的正负异常与持续 $5 \mathrm{~d}$ 及以上 降水频数异常具有较好的一致性.

\section{4 结论}

本文围绕江淮地区梅雨期各类持续性降水, 展 开了各类持续性降水年代际变异的研究, 揭示出 2000 年后长持续时间降水明显减少的事实. 并从低 层大气季节内扰动的角度, 着重探讨持续 $5 \mathrm{~d}$ 及以上 的降水频数年代际变异的可能机制, 得出以下结论.

(1) 气候平均而言, 江淮流域梅雨期多为持续 $2 \mathrm{~d}$ 的降水, 其次则是持续 $3 \sim 4 \mathrm{~d}$, 持续 $5 \mathrm{~d}$ 及以上的降水 过程最少. 并且, 随着持续时间的增加, 总频数逐渐 减少, 并且频发区从江淮的北部地区逐步向南部地 区移动.

（2）不同时期的均方差场表明，在 1980 1999年， 各类持续性降水频数年际差异最大, 尤其是持续 $3 \sim 4 \mathrm{~d}$ 的降水. 然而, 近年来, 各类持续性降水频数的年际 差异均减小, 主要的差异大值区位于江淮地区的东 南部.

（3）分析不同时期的线性趋势发现，持续 $2 \mathrm{~d}$ 的 降水频数在 1960 1979年和 1980 1999年均表现为长 江以南地区减少, 以北则显著增加, 而持续 $5 \mathrm{~d}$ 及以 上降水频数在南部地区为显著的线性上升趋势, 尤 其在 1980 1999 年; 近年来, 持续 $2 \mathrm{~d}$ 降水频数全场 增长, 尤其是长江沿岸和长江人海口, 然而, 持续 $5 \mathrm{~d}$ 及以上降水频数在 $28^{\circ} \sim 30^{\circ} \mathrm{N}$ 之间明显减少.

(4) 持续 $5 \mathrm{~d}$ 及以上降水频数的年代际变异与低 层风场的季节内扰动之间关系明显，尤其是 1990 2007 年. 2000 年后, 持续时间较长的低频风场的单峰 结构已被持续时间较短的多个峰值所取代, 并且持 续性的降水过程发生时间也有所提前. 同时, 不同时 期低层斜压性的增加(减少)与长持续性降水频数的 增加(减少)相吻合. 这都验证了江淮地区持续性较长 的降水在近年来明显减少.

本文的工作只是一个初步的诊断分析工作，与 之相关的数值模式和动力学分析目前还在进行中.

致谢感谢美国国家环境预测中心(NCEP)、欧洲中期预报中心(ECMWF) 和中国气象局国家气象信息中心提供相关气象 资料。 
1 魏风英, 张京江. 1885-2000 年长江中下游梅雨特征量的统计分析. 应用气象学报, 2004, 15: 313-320

2 陈艺敏, 钱永甫. $116 \mathrm{a}$ 长江中下游梅雨的气候特征. 南京气象学院学报, 2004, 27: 65-72

3 徐卫国, 江静. 我国东部梅雨雨区的年际和年代际的变化分析. 南京大学学报(自然科学), 2004, 40: 292-303

4 Wu R G, Wang B. A contrast of the east Asian summer monsoon and ENSO relationship between 1962—1977 and 1978—1993. J Clim, 2002, 15: 3266-3279

5 徐群. 121 年梅雨演变中的近期强年代际变化. 水科学进展, 2007, 18: 327-335

6 梁萍, 何金海. 江淮梅雨气候变化研究进展. 高原气象, 2008, 27: 1-15

7 司东, 丁一汇，刘艳菊. 中国梅雨雨带年代际尺度上的北移及其原因. 科学通报, 2010, 55: 68-73

8 梁萍, 丁一汇. 上海近百年梅雨的气候变化特征. 高原气象, 2008, 27: 76-83

9 黄刚. NCEP/NCAR 和 ERA-40 再分析资料以及探空观测资料分析中国北方地区年代际气候变化. 气候与环境研究，2006，11: $310-320$

$10 \mathrm{Wu}$ R, Kinter J L, Kirtman B P. Discrepancy of interdecadal changes in the Asian region among the NCEP/NCAR reanalysis, objective analyses, and observations. J Clim, 2005, 18: 3048-3067

11 Inoue T, Matsumoto J. A comparison of summer sea level pressure over east Eurasia between NCEP/NCAR reanalysis and ERA-40 for the period of 1960-99. J Meteor Soc Jpn, 2004, 82: 951-958

12 Yang S, Lau K M, Kim K M. Variations of the east Asian jet stream and Asian-Pacific-American winter climate anomalies. J Clim, 2002, 15: $306-325$

13 Hamming R W. Digital Filters. 3rd ed. Hertfordshire: Prentice Hall International (UK) Ltd, 1989. 284-302

14 Yin J H. A consistent poleward shift of the storm tracks in simulations of 21st century climate. Geophys Res Lett, 2005, 32: L18701

15 Lindzen R S, Farrell B F. A simple approximate results for maximum growth rate of baroclinic instabilities. J Atmos Sci, 1980, 60: 14901503

16 Murakami M. Large-scale aspects of deep convective activity over the GATE area. Mon Weather Rev, 1979, 107: 994-1013

$17 \mathrm{Xu}$ Q. Abrupt change of the mid-summer climate in central east China by the influence of atmospheric pollution. Atmos Environ, 2001, 35: $5029-5040$

18 Gong D Y, Ho C H. Shift in the summer rainfall over the Yangtze River valley in the late 1970s. Geophys Res Lett, 2002, $29: 1436$

19 Weng H, Lau K M, Xue Y. Multi-scale summer rainfall variability over China and its long-term link to global sea surface temperature variability. J Meteor Soc Jpn, 1999, 77: 845-857

20 Ding Y, Wang Z, Sun Y. Inter-decadal variation of the summer precipitation in East China and its association with decreasing Asian summer monsoon. Part I : Observed evidences. Int J Climatol, 2007, 28: 1139-1161

21 竺夏英, 何金海, 吴志伟. 江淮梅雨期降水经向非均匀分布及异常年特征分析. 科学通报, 2007, 52: 951一957

22 刘丹妮, 何金海, 姚永红. 关于梅雨研究的回顾与展望. 气象与减灾研究, 2009, 32: 1-9

23 温之平, 董灵英, 吴丽姬, 等. 大气 30-60 d 振荡特征及其与广东持续性强降水的联系. 中山大学学报 (自然科学版), 2007, 46: 98一 103

24 Yang H, Yang C Y. The relation between atmospheric intraseasonal oscillation and summer severe flood and drought in the Changjiang-Huaihe River basin. Adv Atmos Sci, 2003, 20: 540-553

25 韩荣青, 李维京, 董敏. 北半球副热带-中纬度太平洋大气季节内振荡的纬向传播与东亚夏季旱涝. 气象学报, 2006, 64: 149一163

26 孙丹, 琚建华, 吕俊梅. 2003 年东亚季风季节内振荡对我国东部地区降水的影响. 热带气象学报, 2008, 24: 641一648

27 琚建华, 赵尔旭. 东亚夏季风区的低频振荡对长江中下游早涝的影响. 热带气象学报, 2005, 21: 163-171

28 毛江玉, 吴国雄. 1991 年江淮梅雨与副热带高压的低频振荡. 气象学报, 2005, 63: 762-770

29 齐艳军, 张人禾, Tim Li, 等. 大气季节内振荡在印度夏季风建立和年际变化中的作用. 科学通报, 2008, 53: 2972-2975

30 任宏利, 张培群, 丑纪范, 等. 中国夏季大尺度低频雨型及其转换模. 科学通报, 2005, 24: 2790-2799 